\title{
Cellular responses to hypoxia in the pulmonary circulation
}

\author{
Seema O Brij, Andrew J Peacock
}

Oxygen is essential for aerobic respiration to produce ATP. It is possible that all cells are able to sense changes in oxygen content or tension but the mechanisms by which different cell types sense these changes remain poorly understood. The glomus cells of the carotid body, neuroepithelial bodies of the airway mucosa, and the cells of the pulmonary vasculature in animals and humans are fast responding systems and exhibit specific physiological responses when exposed to low oxygen tensions. In addition, certain gene systemserythropoietin (EPO) is the classic examplealso respond to hypoxia. However, the highest levels of EPO are seen in patients with aplastic anaemia, suggesting that EPO upregulation is proportional to oxygen concentration and not oxygen tension.

Alveolar hypoxia causes pulmonary vasoconstriction and, if this hypoxic period is prolonged, there is vascular remodelling which renders the vessel indistensible such that fixed pulmonary hypertension ensues. The processes by which hypoxic pulmonary vasoconstriction and hypoxic vascular remodelling occur need to be fully investigated if the pathological end points are to be reversed in patients exposed to hypoxia, whether secondary to lung disease or to residence at high altitude.

In non-anaesthetised animals the characteristic cardiac and respiratory responses to acute hypoxia are well documented. ${ }^{1-3}$ The respiratory rate, heart rate, and cardiac output all increase to maintain arterial oxygen tension and ensure adequate tissue oxygenation. These responses are thought to be mediated by the peripheral arterial chemoreceptors. In the newborn animal these circulatory and respiratory responses to hypoxia are less developed but by six weeks the adult response is intact. ${ }^{4}$ In the mammalian fetus the high pulmonary vascular tone ensures a right to left shunt of blood through the patent foramen ovale and ductus arteriosus, thereby bypassing the lungs. This active vasomotor response persists in adulthood but only to maximise perfusionventilation matching in the face of regional hypoxia. ${ }^{5}$

Hypoxia decreases the systemic vascular resistance and yet the pulmonary vascular resistance increases. In 1946 Von Euler and Liljestrand demonstrated in the anaesthetised cat that pulmonary artery pressure was inversely proportional to oxygen tension. ${ }^{6} \mathrm{Hyp}-$ oxic pulmonary vasoconstriction (HPV) is intrinsic to the lung and can be demonstrated in isolated perfused lungs, ${ }^{7}$ small pulmonary artery rings, ${ }^{8}$ and in isolated vascular smooth muscle cells. ${ }^{9} 10$ This response is complicated. Due to their elastic and recoil properties the largest proximal arteries act as conduit vessels. Conduit pulmonary arteries have a biphasic response to hypoxia with an initial constriction followed by a sustained relaxation. ${ }^{11}$ The smaller muscularised arteries act as resistance vessels and constrict in response to hypoxia. ${ }^{9}$ This is the response known as hypoxic pulmonary vasoconstriction (HPV). HPV is specific to alveolar hypoxia and does not occur when there is hypoxaemia without alveolar hypoxia ${ }^{12}$ - for example, in a right to left intracardiac shunt.

\section{Hypoxia, oxygen sensing, and vasoconstriction}

Oxygen sensing has been well studied but the mechanism by which oxygen is detected at a cellular level is still uncertain. The smooth muscle cell of the pulmonary vasculature, the glomus cell of the carotid body, and the neuroepithelial bodies of the airway all elicit a fast response to hypoxia. There is increased activity within seconds to minutes: the pulmonary smooth muscle cell constricts, ${ }^{9}{ }^{10}$ the glomus cell releases dopamine, ${ }^{13}$ and there is enhanced secretion of dense core vesicles containing serotonin from neuroepithelial bodies. ${ }^{14}$ The common link in their cellular response to hypoxia is a reduction in whole cell potassium current and a rise in intracellular calcium.

HYPOXIA AND CELLULAR ION CHANNELS

The response of individual ion channels can be studied using patch clamp techniques. In essence, a micro-electrode is placed against the surface of a cell and suction is applied to the electrode creating a high resistance seal. The sealed patch electrode can then be used to monitor the activity of whatever channels happen to be trapped inside the seal. The patch of membrane can be studied in situ or removed from the cell so that the composition of the solution in contact with the membrane may be manipulated. In a similar way whole cell current can also be measured.

Membrane potential is normally maintained between -40 and $-60 \mathrm{mV}$. This transmembrane potential is largely controlled by potassium (K) channels. When $\mathrm{K}$ channels are opened positively charged ions move from the interior to the exterior of the cell. This results in the interior of the cell becoming relatively more negative compared with the exterior. This is membrane hyperpolarisation. If the $\mathrm{K}$ channels are blocked the interior of the cell becomes less negative and membrane depolarisation results.

At least four classes of $\mathrm{K}$ channels have been identified in vascular smooth muscle cells: voltage dependent $\left(\mathrm{K}_{\mathrm{v}}\right)$ of which delayed recti- 
fier $\left(K_{D R}\right)$ channels are a subset, calcium activated $\left(\mathrm{K}_{\mathrm{Ca}}\right)$, ATP sensitive $\left(\mathrm{K}_{\mathrm{ATP}}\right)$, and inward rectifier $\left(\mathrm{K}_{\mathrm{IR}}\right)$ potassium channels. $\mathrm{K}_{\mathrm{V}}$ and $\mathrm{K}_{\mathrm{DR}}$ channels are activated by membrane depolarisation. Once opened, $K_{D R}$ channels restore resting membrane potentials to normal. $\mathrm{K}_{\mathrm{Ca}}$ channels are activated by depolarisation and an increase in intracellular calcium. $\mathrm{K}_{\mathrm{ATP}}$ channels are not voltage dependent and are inhibited by intracellular ATP. When ATP levels fall these channels open and produce hyperpolarisation of the membrane. $\mathrm{K}_{\mathrm{IR}}$ channels control movement of potassium ions from the extracellular milieu into the cell.

Calcium channels also exist in the plasma membrane of cells and are responsible for the influx of calcium ions. Two types of calcium channels have been identified of which the voltage dependent (L-type) is the most extensively studied. The "open state probability" of this channel increases with membrane depolarisation - that is, the more positive the interior of the cell (depolarisation) the more likely the calcium channel is to be open.

The membrane stability and subsequent entry of calcium into a cell is therefore modified by the type and status of the $\mathrm{K}$ channel. The opening of a $\mathrm{K}$ channel which hyperpolarises the membrane reduces the open probability of the voltage gated calcium channel and inhibits calcium influx. In the same way, the closure of a $\mathrm{K}$ channel which depolarises the membrane and increases the open state probability of the calcium channel allows calcium ions to enter the cell.

HYPOXIA AND THE CAROTID BODY

The carotid body is known to sense hypoxia and is responsible for the hyperventilation seen in non-anaesthetised mammals. ${ }^{15}$ These receptors consist of two types of glomus cells (I or II) and are neuroectodermal in origin. Type I (chief) cells contain cytosolic granules rich in dopamine surrounded by type II (sustentacular) cells which are glia-like and are responsible for the carotid body hyperplasia seen in patients with chronic hypoxic lung disease $\mathrm{e}^{16}$ and at altitude. ${ }^{17}$ Carotid bodies are vascular structures for their size and have a small arterial-venous oxygen difference in spite of their high metabolic rate. They respond to changes in arterial oxygen tension but not to anaemia or reduced blood flow. If the carotid bodies are removed the response to hypoxia is depression of respiration via inhibition of the central respiratory centres.

Patch clamp techniques have demonstrated selective inhibition of a delayed rectifier potassium channel in rabbit glomus type I cells when exposed to hypoxia. ${ }^{18}$ The induced membrane depolarisation opens a voltage dependent (L-type) calcium channel which increases cytosolic calcium and facilitates neurotransmitter release. Type I glomus cells synthesise and secrete dopamine during hypoxia. ${ }^{19}$ Activated sensory terminals of afferent sensory fibres of the sinus nerve (a branch of the glossopharyngeal nerve) relay information to respiratory centres in the brainstem and stimulate ventilation.
HYPOXIA AND THE NEUROEPITHELIAL BODIES Pulmonary neuroepithelial bodies are found throughout the airway mucosa in human and animal lungs. ${ }^{14}$ They consist of islands of amine and peptide containing vesicles which are released upon hypoxic stimulation. ${ }^{14}$ These neuroepithelial bodies are therefore similar to other chemoreceptors such as the carotid body and the taste bud. ${ }^{20}$

Patch clamp techniques have demonstrated an oxygen sensitive potassium channel in neuroepithelial body cells. ${ }^{21}$ During hypoxia potassium channels close, inducing membrane depolarisation. Voltage dependent calcium channels are opened which increases cytosolic calcium and facilitates neurotransmitter release. This suggests that neuroepithelial body cells may be transducers of the hypoxic stimulus but their role remains uncertain.

\section{HYPOXIA AND PULMONARY SMOOTH MUSCLE}

CELLS

The mechanism by which the cells of the pulmonary vasculature sense hypoxia is uncertain but there is a likely role for the oxygen sensitive ion channels. In cultured pulmonary artery smooth muscle cells hypoxia alters potassium channel activity in a similar fashion to that seen in the type I cell of the carotid body and neuroepithelial bodies. ${ }^{22}{ }^{23}$ This reduction in whole cell potassium current may provoke membrane depolarisation and calcium influx. Calcium influx has been shown to initiate pulmonary smooth muscle cell contraction ${ }^{24}$ which is clinically manifest as hypoxic pulmonary vasoconstriction.

Systemic vascular smooth muscle cells from cerebral, ${ }^{9}$ mesenteric, ${ }^{25}$ and renal ${ }^{23}$ circulations do not inhibit potassium current in response to hypoxia. In systemic circulations exposed to hypoxia it is thought that $\mathrm{K}_{\mathrm{ATP}}$ channels are opened, thereby hyperpolarising the membrane and inhibiting calcium influx. ${ }^{26}$ Thus there is smooth muscle cell relaxation which is clinically manifest as systemic vasodilatation.

In the pulmonary artery the distribution of $\mathrm{K}$ channel subtypes in small distal resistance arteries differs from those in the larger proximal conduit arteries. ${ }^{27}$ Resistance artery smooth muscle cells have a higher density of $\mathrm{K}_{\mathrm{DR}}$ rectifier potassium channels which exhibit oxygen sensitivity by their $\mathrm{K}$ channel inhibition. Conversely, in the conduit vessels there is a higher proportion of $\mathrm{K}_{\mathrm{Ca}}$ channels which increase whole cell potassium current on hypoxic exposure. Conduit and resistance myocytes also differ in their voltage gated (L-type) calcium channel response to hypoxia. In the conduit arteries hypoxia inhibits calcium current whereas in resistance arteries hypoxia increases calcium influx. ${ }^{28}$

The heterogeneity in distribution of $\mathrm{K}$ and calcium channels may explain some of the discrepancies in vascular reactivity noted between resistance and conduit arteries in response to hypoxia. Resistance arteries respond to hypoxia ${ }^{9}$ by inhibition of whole cell potassium current and depolarisation of the membrane leading to calcium influx and constriction. Conduit arteries are more akin to systemic 


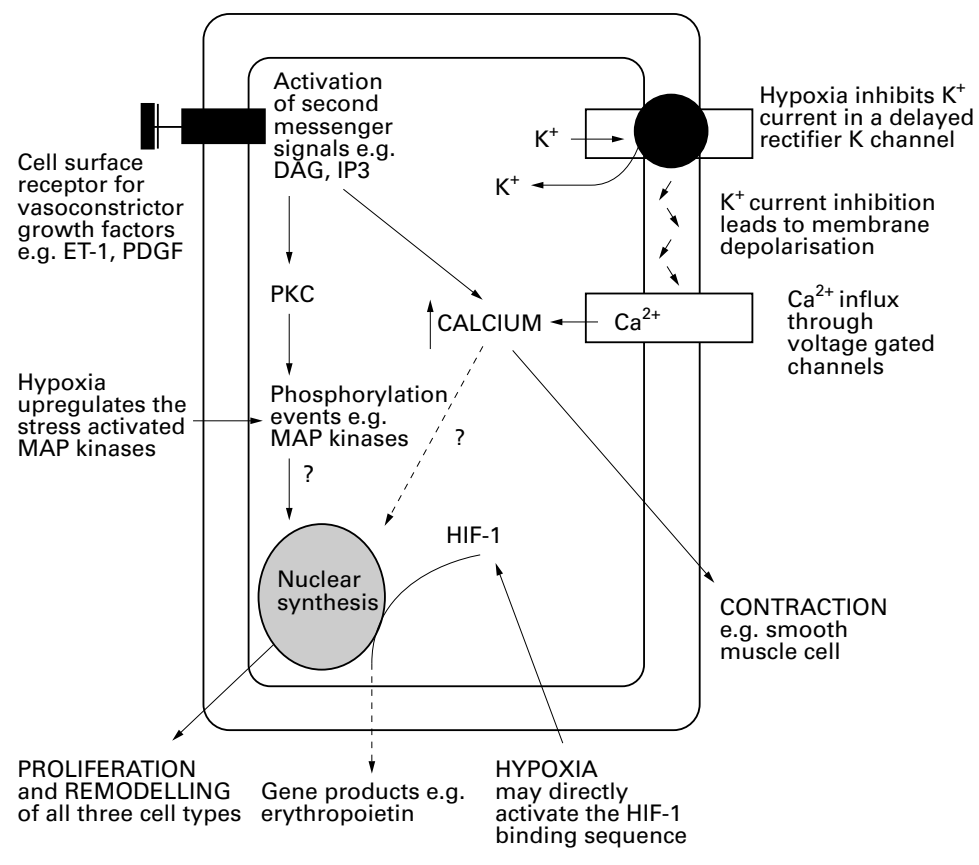

Figure 1 Cellular responses to hypoxia. (1) Hypoxia inhibits $K^{+}$current in an oxygen sensitive potassium channel (delayed rectifier type) which leads to membrane depolarisation and subsequent $\mathrm{Ca}^{2+}$ influx through voltage gated calcium channels. The rise in intracellular calcium is sufficient to induce smooth muscle contraction. (2) Hypoxia may attenuate the effects of endothelin 1 (ET-1) and platelet-derived growth factor (PDGF) in the pulmonary circulation. Second messengers in the cytosol are activated when the respective receptor sites are occupied. The hydrolysis of phosphatidylinositol-4,5-bisphosphate (PIP,) liberates diacylglycerol $(D A G)$ and inositol-1,4,5-triphosphate $\left(I P_{3}\right) . D A G$ activates protein kinase $C(P K C)$ which is upstream to stress response mitogen activated protein kinases (MAP kinases) in intracellular proliferative signalling cascades. (3) Hypoxia upregulates the stress activated MAP kinases. (4) Hypoxia causes an increase in nuclear transcription which may be regulated by a hypoxia inducible factor (HIF-1). HIF-1 also regulates other hypoxia induced genes such as erythropoietin.

arteries and respond to hypoxia by potentiation of potassium current and hyperpolarisation of the membrane with no resulting increase in cytosolic calcium and therefore relaxation. ${ }^{11}$

\section{Pulmonary vascular cell proliferation and} hypoxia

The rise in intracellular calcium is thought to activate the cellular mechanisms which result not only in pulmonary smooth muscle cell contraction $^{24}$ but also in the proliferative response and remodelling which occurs in all three layers in the vessel wall in response to hypoxia.

THE HYPOXIC PROLIFERATIVE RESPONSE

Chronic hypoxia is characterised by a sustained increase in pulmonary vascular tone which in part is maintained by vascular remodelling. The duration of the hypoxia, its severity, and the time of life at which the exposure is experienced all serve to modify the outcome. In addition, each cell type in the pulmonary arterial wall - the endothelial cell, the smooth muscle cell and the adventitial fibroblast-has a specific growth response to hypoxia.

Meyrick and Reid ${ }^{29}$ found that within 24 hours of hypoxia in vivo there was an increase in proliferation of the endothelial cell, medial smooth muscle cell, and adventitial fibroblast. However, the hypoxic proliferative response was different in proximal hilar vessels and distal intra-alveolar vessels. In the hilar vessels hypoxia caused a threefold increase in endothe- lial cell proliferation. There was minimal change in the medial smooth muscle in comparison with the adventitial fibroblasts which showed an eightfold increase in proliferation. In small intra-alveolar arteries hypoxia stimulated smooth muscle cell proliferation and the muscularisation of previously nonmuscular small arteries. The endothelial cell and adventitial fibroblasts also showed increased proliferation, though not as striking as in the proximal vessels. ${ }^{29}$

The adventitial fibroblast is unique in that it proliferates directly in response to hypoxia. ${ }^{30}$ This response is augmented with the addition of growth factors. ${ }^{31}$ Marked increases in fibroblastic matrix deposition-namely, collagen and elastin-has been observed under conditions of chronic hypoxia ${ }^{32}$ which contribute to the indistensibility of the vessel wall.

\section{HYPOXIC PROLIFERATION AND INTRACELLULAR} SIGNALLING

Protein kinase C (PKC) is a family of intracellular enzymes known to play a significant role in the signal transduction of normal cellular proliferation. PKC is a calcium dependent enzyme and, once activated, it stimulates DNA synthesis in systemic artery smooth muscle cells ${ }^{33}$ and influences $\mathrm{Na}^{+}-\mathrm{H}^{+}$ exchange. ${ }^{34}$

PKC is known to be important in the growth of pulmonary vascular cells. Pulmonary myocytes will not proliferate in response to hypoxia unless PKC is activated..$^{35}$ Cultured fetal and neonatal pulmonary adventitial fibroblasts exhibit increased expression of PKC during hypoxic growth (fig 1 ). ${ }^{36}$

PKC is also activated by the lipid second messenger diacylglycerol which is generated by the hydrolysis of phosphatidylinositol-4,5bisphosphate $\left(\mathrm{PIP}_{2}\right)$. The other by-product of this reaction, inositol-1,4,5-triphosphate $\left(\mathrm{IP}_{3}\right)$, translocates to a receptor on the endoplasmic reticulum triggering the release of intracellular calcium. Hypoxia enhances the proliferation and generation of $\mathrm{IP}_{3}$ in pulmonary artery fibroblasts but not in those from the mesenteric circulation (fig 1). ${ }^{37}$

The mitogen activated protein kinase (MAP kinase) pathway is also important in the signal transduction of normal proliferation and exerts its effects downstream of PKC. MAP kinases are activated by multiple phosphorylation events. A major advance in the understanding of intracellular signalling events in hypoxia is that heat shock, osmotic shock, antioxidants, ultraviolet light and DNA damaging agents are able to activate MAP kinases. Work in our laboratory has shown that hypoxia also stimulates the "stress activated" MAP kinases" which may therefore be involved in the hypoxic proliferative response.

\section{HYPOXIA AND THE UPREGULATION OF GROWTH} MITOGENS

Hypoxia increases the proliferation of all three cell types in the pulmonary arterial wall. Many of the growth factors which are known to cause proliferation in pulmonary vascular cells are also vasoconstrictors - for example, endothelin 
(ET), platelet-derived growth factor (PDGF), vascular endothelial growth factor (VEGF), and serotonin (5-HT). Increased expression of PDGF, ${ }^{39} \mathrm{VEGF}^{40}$ and ET-1 $\mathrm{mRNA}^{41}{ }^{42}$ has been found in hypoxic endothelial cells. Endothelin is a potent vasoconstrictor and is also a mitogen for smooth muscle cells and fibroblasts. ${ }^{43} 44$

HYPOXIA AND UPREGULATION OF OXYGEN SENSITIVE GENES

Hypoxia is known to upregulate several gene system mechanisms. The erythropoietin (Epo) gene is directly stimulated causing increased red cell mass and therefore increased oxygen carrying capacity. ${ }^{45}$ There is a sequence in the Epo gene that confers hypoxia responsiveness. ${ }^{46}$ This area contains a highly conserved 8 base pair sequence (5'TACGTGCT-3') that binds the hypoxia inducible factor-1 (HIF-1) transcription factor.

Hypoxia activates HIF-1 binding activity and the HIF-1 DNA binding element has been found in other genes that are upregulated in response to hypoxia. VEGF is expressed in nearly all cells and tissues and is important in the regulation of new blood vessel formation. The expression of VEGF is markedly increased by hypoxia. ${ }^{40}$ In the VEGF gene there is a $90 \%$ homologous site to a region within the human Epo 3' enhancer. ${ }^{47}$ The hypoxic induction of VEGF also depends on the activation of the HIF-1 transcription factor.

During hypoxia the glomus cells synthesise and release dopamine induced by membrane depolarisation as discussed earlier. Tyrosine kinase $(\mathrm{TH})$ is the rate limiting enzyme in the biosynthesis of dopamine and is stimulated by hypoxia. ${ }^{19} \mathrm{TH}$ mRNA expression increases during hypoxia ${ }^{48}$ and there is a region in the $\mathrm{TH}$ gene which also contains a complementary HIF-1 sequence. ${ }^{49}$

Thus, activation of HIF-1 may be the common mechanism by which Epo, VEGF, and $\mathrm{TH}$ are activated during hypoxia. Other oxygen sensitive genes that are regulated via HIF-1 include the glucose transporter GLUT-1, lactate dehydrogenase, heme oxygenase-1, and inducible nitric oxide synthase (iNOS). The variety of genes sharing a common mechanism of activation suggests a co-ordinated response to the hypoxic stimulus.

\section{Conclusion}

Similarities in the oxygen sensing mechanisms exist in the carotid body, the neuroepithelial body, and pulmonary resistance artery smooth muscle cells. It is the resulting increase in intracellular calcium which initiates hypoxic pulmonary vasoconstriction and proliferation of each cell type. The signalling cascades which upregulate growth factors and increase mRNA levels and drive remodelling and proliferation are as yet unknown.

Hypoxia exerts its effects at the cell surface, in the cytosol, and in the nucleus. Responses to hypoxia have been studied in the whole animal, the isolated organs, the single cell, and at the molecular level. At present there are important isolated pieces of evidence but the whole jigsaw is far from complete. How these cellular processes result in the physiological end points observed, the respiratory and cardiovascular responses, the hypoxic pulmonary vasoconstriction, and pulmonary vascular remodelling is still being investigated. Clearly, a better understanding of the effects of hypoxia is needed if the development of pulmonary hypertension in subjects with hypoxic lung disease or those living at altitude is to be prevented.

1 Baugh CW, Cornatt RW, Hatcher JD. The adrenal gland and the cardiovascular changes in acute anoxic anoxia in dogs. Circ Res 1959; 7:513-20.

2 Gorlin R, Lewis BM. Circulatory adjustments to hypoxia in dogs. f Appl Phsiol 1954;7:180-5.

3 Harrison TR, Blalock A. The regulation of the circulation. VI. The effects of severe anoxaemia of short duration on the cardiac output of morphinised and trained unnarcothe cardiac output of morphinised and t
tized dogs. Am $\mathcal{7}$ Physiol 1927;80:169-78.

4 Sidi D, Kuipers JRG, Teitel D, et al. Developmental changes in oxygenation and circulatory responses to hypoxaemia in in oxygenation and circulatory responses

5 Weir EK, Archer SL. The mechanism of acute pulmonary vasoconstriction: the tale of two channels. FASEB 1995;9: 183-9.

6 Von Euler US, Liljestrand G. Observations on the pulmonary arterial blood pressure in the cat. Acta Physiol Scand 1946;12:301-20.

7 McMurtry I, Davidson A, Reeves J, et al. Inhibition of hypoxic pulmonary vasoconstriction by calcium antagonists in isolated rat lungs. Circ Res 1976;38:99-104.

8 Maden J, Dawson C, Harder D. Hypoxia-induced activation in small isolated pulmonary arteries from the cat. $\mathcal{F} A p p l$ Physiol 1985;59:113-8.

9 Madden JA, Vadula MS, Kurup VP. Effects of hypoxia and other vasoactive agents on pulmonary and cerebral artery smooth muscle cells. Lung Cell Mol Physiol 1992; 7:L38493.

10 Vadula MS, Kleinman JG, Madden JA. Effects of hypoxia and norepinephrine on cytoplasmic free $\mathrm{Ca}^{2+}$ in pulmonary and cerebral arterial myocytes. Am f Physiol 1993;265: L591-7.

11 Bennie RE, Packer CS, Powell DR, et al. Biphasic contractile response of pulmonary artery to hypoxia. Am $\mathcal{F}$ Physiol 1991;263:L156-63.

12 Lloyd TC. Pulmonary vasoconstriction during histotoxic hypoxia. $\mathcal{f}$ Appl Physiol 1965;20:488-90.

13 Fidone S, Gonzalez C, Yoshizaki K. Effects of low oxygen on the release of dopamine from the rabbit carotid body in vitro. F Physiol 1982;333:93-110.

14 Lauweryns JM, Cokelaere $\mathrm{M}$, Deleersynder $\mathrm{M}$, et al. Intrapulmonary neuroepithelial bodies in newborn rabbits: influence of hypoxia, hyperoxia, hypercapnia, nicotine, reserpine, L-DOPA and 5-HT. Cell Tissue Res 1977;182: 425-40.

15 Daly MD, Scott MJ. The cardiovascular responses to stimulation of the carotid body chemoreceptors in the dog. 7 Physiol (London) 1963;165:179-97.

16 Heath D. Carotid body hyperplasia. In: Heath D, ed. Aspects of hypoxia. Liverpool: Liverpool University Press, 1986: 61-74

17 Arias-Stella J, Topilsky M. Human carotid body at altitudes. Am f Pathol 1969;55:82(abstract)

8 Ganfornina MD, Lopez-Barneo J. Single $\mathrm{K}^{+}$channels in membrane patches of arterial chemoreceptor cells are modulated by $\mathrm{O}_{2}$ tension. Proc Natl Acad Sci USA 1991;88: 2927-30.

19 Gonzalez C, Kwok Y, Gibb J, et al. Effects of hypoxia on tyrosine hydroxylase activity in rat carotid body. $\mathcal{F}$ Neurochem 1979;33:713-9.

20 Lauweryns JM, Van Lommel A. Effects of various vagotomy procedures on the reaction to hypoxia of rabbit neuroepithelial bodies: modulation by intrapulmonary axon reflexes? Exp Lung Res 1986;11:319-39.

21 Youngson C, Nurse C, Yeger $\mathrm{H}$, et al. Oxygen sensing in airway chemoreceptors. Nature 1993;365:153-5.

22 Yuan X-J, Tod M, Rubin L, et al. Contrasting effects of Yuan X-J, Tod M, Rubin L, et al. Contrasting effects of
hypoxia on tension in rat pulmonary and mesenteric arterhypoxia on tension in rat pulmonary

23 Post J, Hume J, Archer SL, et al. Direct role for potassium channel inhibition in hypoxic pulmonary vasoconstriction. Am F Physiol 1992;262:H882-90.

24 Murray TR, Chen L, Marshall BE, et al. Hypoxic contraction of cultured pulmonary vascular smooth muscle cells. Am $\mathcal{F}$ Respir Cell Mol Biol 1990;3:457-65.

25 Yuan X-J, Goldman WF, Tod M, et al. Hypoxia reduces potassium currents in cultured rat pulmonary but not mesenteric arterial myocytes. Am F Physiol 1993;264:L11623.

26 Daut J, Maier-Rudolph W, von Beckerath N, et al. Hypoxic dilation of coronary arteries is mediated by ATP-sensitive potassium channels. Science 1990;247:1341-4.

27 Archer SL, Huang JMC, Reeve HL, et al. Differential distribution of electrophysiologically distinct myocytes in conduit and resistance arteries determines their response to nitric oxide and hypoxia. Circ Res 1996;78:431-42.

28 Franco-Obregon A, Lopez-Barneo J. Differential oxygen sensitivity of calcium channels in rabbit smooth muscle 
cells of conduit and resistance pulmonary arteries. $\mathcal{F}$ Physiol (London) 1996;491:511-8.

29 Meyrick B, Reid L. Hypoxia and incorporation of $[3 \mathrm{H}]$-thymidine by cells of the rat pulmonary arteries and (1979;96:51-70

30 Storch TJ, Talley GD. Oxygen concentration regulates the proliferative response of human fibroblasts to serum and growth factors. Exp Cell Res 1988;175:317-25.

31 Peacock AJ, Dawes KE, Shock A, et al. Endothelin-1 and endothelin-3 induce chemotaxis and replication of pulmonary artery fibroblasts. Am 7 Respir Cell $\mathrm{Mol}$ Bio 1992;7:492-9.

32 Durmowicz AG, Parks WC, Hyde DM, et al. Persistence, re-expression and induction of pulmonary arterial fibronectin, tropoelastin and type I procollagen mRNA expression in neonatal hypoxic pulmonary hypertension. $A m \mathcal{F}$ Pathol 1994;145:1411-20.

33 Owen NE. Effect of TPA on ion fluxes and DNA synthesis in vascular smooth muscle cells. F Cell Biol 1985;101:454-9.

34 Berk BC, Brock TA, Gimbrone MAJ, et al. Early agonist-mediated ionic events in cultured vascular smooth muscle cells. F Biol Chem 1987;262:5056-72.

35 Dempsey EC, McMurtry IF, O'Brien RF. Protein kinase C activation allows pulmonary artery smooth muscle cells to activation allows pulmonary artery smooth muscle cells

36 Das M, Stenmark KR, Dempsey EC. Enhanced growth of fetal and neonatal pulmonary artery adventitial fibroblasts is dependent on protein kinase C. Am f Physiol 1995;269: L660-7.

37 Welsh DJ, Scott P, Plevin R, et al. Hypoxia enhances cellular proliferation and inositol 1,4,5-trisphosphate generation in fibroblasts from bovine pulmonary artery but not from mesenteric artery. Am f Respir Crit Care Med 1998(in press)

38 Scott PH, Paul A, Belham CM, et al. Hypoxic stimulation of the stress-activated protein kinases in pulmonary artery fibroblasts. Am $\mathcal{F}$ Respir Crit Care Med 1998(in press).
39 Katayose D, Masatoshi O, Yamauchi $\mathrm{K}$, et al. Increased expression of PDGF A- and B-chain genes in rat lungs with hypoxic pulmonary hypertension. Am f Physiol 1993;264: L100-6.

40 Shweiki D, Neeman M, Itin A, et al. Vascular endothelial growth factor induced by hypoxia may mediate hypoxiainitiated angiogenesis. Nature 1992;359:843-5.

41 Stelzner TJ, O'Brien RF, Yanagisawa M, et al. Inreased lung endothelial-1 production in rats with idiopathic pulmonary hypertension. Am F Physiol 1992;262:L614-20.

42 Kourembanas S, Marsden PA, McQuillan LP, et al. Hypoxia induces endothelin gene expression and secretion in cultured human endothelium. F Clin Invest 1991;88:1054-7.

43 Komuro I, Kurihara H, Sugiyama T, et al. Endothelin stimulates c-fos and c-myc expression and proliferation of vascular smooth muscle cells. FEBS 1988;238:249-52.

44 Nakaki $T$, Nakayama $M$, Yamamoto $S$, et al. Endothelin stimulation of DNA synthesis in vascular smooth muscle cells. Biochem Biophys Res Commun 1989;158:880-3.

45 Beru S, McDonald J, Lacombe C, et al. Expression of the erythropoietin gene. Mol Cell Biol 1986;6:2571-5.

46 Semenza GL, Wang GL. A nuclear factor induced by hypoxia via de novo protein synthesis binds to human erythropoietin gene enhancer at a site required for transcriptional activation. Mol Cell Biol 1992;12:5447-54.

47 Goldberg MA, Schneider TJ. Similarities between the oxygen-sensing mechanisms regulating the expression of vascular endothelial growth factor and erythropoietin. $\mathcal{f}$ Biol Chem 1994;269:4355-9.

48 Czyzyk-Krzeska MF, Furnari B, Lawson E, et al. Regulation of tyrosine hydroxylase gene expression in the rat carotid body by hypoxia. F Neurochem 1992;58:1538-46.

49 Norris ML, Millhorn DE. Hypoxia-induced proteinbinding to $\mathrm{O}_{2}$-responsive sequences on the tyrosine hydroxylase gene. $\mathcal{F}$ Biol Chem 1995;270:23774-9. 